Korean J. Math. 20 (2012), No. 4, pp. 371-379

http://dx.doi.org/10.11568/kjm.2012.20.4.371

\title{
KRONECKER FUNCTION RINGS AND PRÜFER-LIKE DOMAINS
}

\author{
Gyu Whan Chang
}

\begin{abstract}
Let $D$ be an integral domain, $\bar{D}$ be the integral closure of $D, *$ be a star operation of finite character on $D, *_{w}$ be the so-called $* w_{\text {-operation }}$ on $D$ induced by $*, X$ be an indeterminate over $D, N_{*}=\left\{f \in D[X] \mid c(f)^{*}=D\right\}$, and $\operatorname{Kr}(D, *)=$ $\{0\} \cup\left\{\frac{f}{g} \mid 0 \neq f, g \in D[X]\right.$ and there is an $0 \neq h \in D[X]$ such that $\left.(c(f) c(h))^{*} \subseteq(c(g) c(h))^{*}\right\}$. In this paper, we show that $D$ is a $*$-quasi-Prüfer domain if and only if $\bar{D}[X]_{N_{*}}=K r\left(D, *_{w}\right)$. As a corollary, we recover Fontana-Jara-Santos's result that $D$ is a Prüfer *-multiplication domain if and only if $D[X]_{N_{*}}=K r\left(D, *_{w}\right)$.
\end{abstract}

\section{Introduction}

Let $D$ be an integral domain with quotient field $K, \bar{D}$ be the integral closure of $D$ in $K, X$ be an indeterminate over $D$, and $D[X]$ be the polynomial ring over $D$. For any $f \in D[X]$, we denote by $c_{D}(f)$ (simply $c(f)$ ) the ideal of $D$ generated by the coefficients of $f$. For an ideal $A$ of $D[X]$, let $c_{D}(A)=\sum_{f \in A} c(f)\left(\operatorname{simply} c_{D}(A)\right.$ is denoted by $\left.c(A)\right)$.

Let $*$ be a star operation on $D$. (Definitions related to star operations will be reviewed in the sequel.) Recall that $D$ is a Prüfer $*$-multiplication

Received March 31, 2012. Revised October 10, 2012. Accepted October 15, 2012.

2010 Mathematics Subject Classification: 13 A15.

Key words and phrases: Star operation, Kronecker function ring, *-quasi-Prüfer domain.

This work was supported by Basic Science Research Program through the National Research Foundation of Korea (NRF) funded by the Ministry of Education, Science and Technology (2010-0007069).

(C) The Kangwon-Kyungki Mathematical Society, 2012.

This is an Open Access article distributed under the terms of the Creative commons Attribution Non-Conercial License (http://creativecommons.org/licenses/bync/3.0/) which permits unrestricted non-commercial use, distribution and reproduction in any medium, provided the original work is properly cited. 
domain $(\mathrm{P} * \mathrm{MD})$ if each nonzero finitely generated ideal $I$ of $D$ is $*_{f^{-}}$ invertible, i.e., $\left(I I^{-1}\right)^{* f}=D$. A nonzero prime ideal $Q$ of $D[X]$ is an upper to zero in $D[X]$ if $Q \cap D=(0)$. As in [5], we say that $D$ is $*_{f}$-quasi-Prüfer if every upper to zero in $D[X]$ contains an $f \in D[X]$ with $c_{D}(f)^{*_{f}}=D$. It is known that $D$ is a $\mathrm{P} * \mathrm{MD}$ if and only if $D$ is an integrally closed $*_{f}$-quasi-Prüfer domain [14, Theorem 1.1]. Moreover, $D$ is $d$-quasi-Prüfer if and only if $\bar{D}$ is a Prüfer domain [8, Corollary 6.5.14].

Let $*_{c}$ be the e.a.b. star operation on an integrally closed domain $D$ induced by $*$ (see Lemma 1$)$, and let $K r\left(D, *_{c}\right)$ be the Kronecker function ring of $D$ with respect to $*_{c}$. It is known that $D$ is a $\mathrm{P} * \mathrm{MD}$ if and only if $\operatorname{Kr}\left(D, *_{c}\right)=D[X]_{N_{*}}$, where $N_{*}=\left\{f \in D[X] \mid c_{D}(f)^{*}=D\right\}$, [4, Theorem 3.7]. This result provides a generalization of $[2$, Theorem 4] that $D$ is a Prüfer domain if and only if $D(X)=K r(D, b)$, where $D(X)=\left\{\frac{f}{g} \mid f, g \in D[X], 0 \neq g\right.$ and $\left.c(g)=D\right\}$. In [10], Fontana-Loper used an arbitrary star operation to define the Kronecker function ring (see Lemma 2). Using this notion of Kronecker function rings, in [9, Theorem 3.1], Fontana-Jara-Santos showed that $D$ is a $\mathrm{P} * \mathrm{MD}$ if and only if $D[X]_{N_{*}}=\operatorname{Kr}\left(D, *_{w}\right)$.

In this paper, we also use this Kronecker function ring to characterize $*_{f}$-quasi-Prüfer domains. Precisely, we show that $D$ is a $*_{f}$-quasi-Prüfer domain if and only if $\bar{D}[X]_{N_{*}}=K r\left(D, *_{w}\right)$. As a corollary, we recover Fontana-Jara-Santos's result $\left[9\right.$, Theorem 3.1], because $D[X]_{N_{*}} \cap K=D$ and $\operatorname{Kr}\left(D, *_{w}\right)$ is integrally closed.

We next review some definitions and notations related to star operations. Let $\mathbf{F}(D)$ (resp., $\mathbf{f}(D)$ ) be the set of nonzero fractional (resp., nonzero finitely generated fractional) ideals of $D$. A mapping $I \mapsto I^{*}$ of $\mathbf{F}(D)$ into $\mathbf{F}(D)$ is called a star operation on $D$ if the following three conditions are satisfied for all $0 \neq a \in K$ and $I, J \in \mathbf{F}(D)$ :

(1) $(a D)^{*}=a D$ and $(a I)^{*}=a I^{*}$,

(2) $I \subseteq I^{*} ; I \subseteq J$ implies $I^{*} \subseteq J^{*}$ and

(3) $\left(I^{*}\right)^{*}=I^{*}$.

It is well known that the mapping $I \mapsto I^{*_{f}}=\cup\left\{J^{*} \mid J \subseteq I\right.$ and $J \in \mathbf{f}(D)\}$ is a star operation on $D$. The $*_{w}$-operation is a star operation on $D$ defined by setting $I^{* w}=\{x \in K \mid x J \subseteq I$ for some $J \in \mathbf{f}(D)$ with $\left.J^{*}=D\right\}$ for all $I \in \mathbf{F}(D)$. A star operation $*$ on $D$ is said to be of finite character if $*_{f}=*$. Clearly, $\left(*_{f}\right)_{f}=*_{f}$ and $*_{w}=\left(*_{f}\right)_{w}=\left(*_{w}\right)_{f}$; so $*_{f}$ and $*_{w}$ are of finite character. The most well-known examples of 
star operations are the $d_{-}, v-, t$-, and $w$-operations. The $d$-operation is just the identity function on $\mathbf{F}(D)$; so $d=d_{f}=d_{w}$. The $v$-operation is defined by $I^{v}=\left(I^{-1}\right)^{-1}$, where $I^{-1}=\{x \in K \mid x I \subseteq D\}$, while the $t$-operation (resp., $w$-operation) is defined by $t=v_{f}$ (resp., $w=v_{w}$ ).

An $I \in \mathbf{F}(D)$ is called a $*$-ideal if $I^{*}=I$. A $*$-ideal is called a maximal $*$-ideal if it is maximal among the proper integral $*$-ideals of $D$. Let $*-\operatorname{Max}(D)$ denote the set of maximal $*$-ideals of $D$. It is well known that a maximal $*_{f}$-ideal is a prime ideal; each integral $*_{f}$-ideal is contained in a maximal $*_{f}$-ideal; $*_{f}-\operatorname{Max}(D) \neq \emptyset$ if $D$ is not a field; and $*_{f}-\operatorname{Max}(D)=*_{w}-\operatorname{Max}(D)[1$, Theorem 2.16]. An $I \in \mathbf{F}(D)$ is said to be $*$-invertible if $\left(I I^{-1}\right)^{*}=D$. Clearly, $I \in \mathbf{F}(D)$ is $* f^{\text {-invertible if and }}$ only if $I I^{-1} \nsubseteq P$ for all $P \in *_{f^{-}} \operatorname{Max}(D)$. As in [3, page 224], we say that an overring $R$ of $D$ is $*$-linked over $D$ if $I^{*}=D$ implies $(I R)^{v}=R$ for all $I \in \mathbf{f}(D)$. A valuation overring $V$ of $D$ is a $*$-valuation overring of $D$ if $I^{*} \subseteq I V$ for all $I \in \mathbf{f}(D)$. Obviously, *-valuation overrings of $D$ are *-linked over $D$, but $*$-linked vauation overrings need not be $*$-valuation overrings (see the paragraph after Lemma 1).

For any two star-operations $*_{1}, *_{2}$ on $D$, we mean by $*_{1} \leq *_{2}$ that $I^{*_{1}} \subseteq I^{*_{2}}$ for all $I \in \mathbf{F}(D)$. We know that if $*_{1} \leq *_{2}$, then $\left(*_{1}\right)_{f} \leq\left(*_{2}\right)_{f}$ and $\left(*_{1}\right)_{w} \leq\left(*_{2}\right)_{w}$. Also, $*_{w} \leq *_{f} \leq *$ and $d \leq * \leq v$ for any star operation $*$ on $D$; hence $d \leq *_{f} \leq t$ and $d \leq *_{w} \leq w$. Clearly, each $t$-ideal is a $*_{f}$-ideal, and thus each maximal $*_{f}$-ideal is a $t$-ideal if and only if $*_{w}=w$. For more on basic properties of star operations, see [3], [11], or [13, Sections 32 and 34].

\section{Kronecker function rings}

Let $D$ be an integral domain with quotient field $K$. A star operation * on $D$ is said to be endlich arithmetisch brauchbar (e.a.b.) if, for all $A, B, C \in \mathbf{f}(D),(A B)^{*} \subseteq(A C)^{*}$ implies $B^{*} \subseteq C^{*}$. Obviously, $*$ is an e.a.b. star operation if and only if $*_{f}$ is an e.a.b. star operation. Let $*$ be an e.a.b. star operation on $D$. The Kronecker function ring of $D$ with respect to $*$ is an integral domain

$$
\operatorname{Kr}(D, *)=\left\{\frac{f}{g} \mid f, g \in D[X], g \neq 0, \text { and } c(f) \subseteq c(g)^{*}\right\} .
$$

It is well known that $\operatorname{Kr}(D, *)$ is a Bezout domain and $\operatorname{Kr}(D, *) \cap K=D$ [13, Theorem 32.7]. Hence if $D$ admits an e.a.b. star-operation, then $D$ 
is integrally closed [13, Corollary 32.8]. Conversely, if $D$ is integrally closed, then the $b$-operation on $D$ defined by $I^{b}=\cap\{I V \mid V$ is a valuation overring of $D\}$ for all $I \in \mathbf{F}(D)$ is an e.a.b. star operation of finite character on $D$ such that $b \leq *$ for any e.a.b. star operation $*$ on $D[13$, Theorem 32.7 and Corollary 32.14]. More generally, we have

Lemma 1. ([4, Lemma 3.1]). Let $D$ be an integrally closed domain and $\left\{V_{\alpha}\right\}$ be the set of $*$-linked valuation overrings of $D$. Then the map $*_{c}: \mathbf{F}(D) \rightarrow \mathbf{F}(D)$, given by $I \mapsto I^{*_{c}}=\cap_{\alpha} I V_{\alpha}$, is an e.a.b. star operation of finite character on $D$ such that $*_{w}=\left(*_{c}\right)_{w} \leq *_{c}$ and $*_{f}-\operatorname{Max}(D)=*_{c^{-}}$ $\operatorname{Max}(D)$. In particular, $d_{c}=b$.

We now give an example of $*$-linked valuation overrings that are not *-valuation overrings. Let $X, y$ be indeterminates over the field $\mathbb{Q}$ of rational numbers, $K=\mathbb{Q}(y), V=K \llbracket X \rrbracket$ be the power series ring, and $D=\mathbb{Q}+X K \llbracket X \rrbracket$. Clearly, $D$ is an integrally closed quasi-local domain whose maximal ideal is a $v$-ideal, and hence each overring of $D$ is $t$-linked over $D$. If every valuation overring $V$ of $D$ is a $t$-valuation overring, then $I^{t} \subseteq I V$, and so $I^{t} \subseteq \cap\{I V \mid V$ is a valuation overring of $D\}=I^{b}$ for all $I \in \mathbf{f}(D)$. Hence $v_{f}=t=b$ because $b \leq t$, and so $v$ is an e.a.b. star operation on $D$. Thus every $I \in \mathbf{f}(D)$ is $v$-invertible [13, Theorem 34.6], and since the maximal ideal of $D$ is a $v$-ideal, $I$ is invertible. But if we let $I=(X, y X)$, then $I$ is not invertible, a contradiction. Therefore there is a ( $t$-linked) valuation overring of $D$ that is not a $t$-valuation overring.

Let $*$ be a star operation on $D$. An $x \in K$ is said to be $*$-integral over $D$ if $x J^{*} \subseteq J^{*}$ for some $J \in \mathbf{f}(D)$. Let $D^{[*]}=\{x \in K \mid x$ is $*$-integral over $D$ \}; then $D^{[*]}$, called the *-integral closure of $D$, is an integrally closed overring of $D$ [17, Theorems 2.3 and 2.8]. We say that $D$ is $*$-integrally closed if $D^{[*]}=D$. In [10], Fontana and Loper used an arbitrary star operation to define a Kronecker function ring.

Lemma 2. ([10, Theorem 5.1, Proposition 4.5(2), and Corollary 3.5]) Let $*$ be a star operation on $D$, and let $\operatorname{Kr}(D, *)=\{0\} \cup\left\{\frac{f}{g} \mid 0 \neq\right.$ $f, g \in D[X]$ and there is an $0 \neq h \in D[X]$ such that $(c(f) c(h))^{*} \subseteq$ $\left.(c(g) c(h))^{*}\right\}$. Then $K r(D, *)$ is a Bezout domain with quotient field $K(X)$ and $K r(D, *) \cap K=D^{[*]}$.

Clearly, if $*$ is e.a.b., then the $K r(D, *)$ of Lemma 2 is the usual Kronecker function ring (so we use the same notation $\operatorname{Kr}(D, *)$ ). It is clear that $K r(D, *)=K r\left(D, *_{f}\right)$ and if $*_{1} \leq *_{2}$ are star operations on $D$, then $\operatorname{Kr}\left(D, *_{1}\right) \subseteq K r\left(D, *_{2}\right)$; in particular, $\operatorname{Kr}(D, d) \subseteq K r(D, w) \subseteq$ 
$\operatorname{Kr}(D, t)=\operatorname{Kr}(D, v)$. For more on $\operatorname{Kr}(D, *)$, see Fontana-Loper's interesting survey article [12].

Assume that $D$ is $*$-integrally closed, and let $I^{*_{a}}=\cup\{J \operatorname{Kr}(D, *) \cap$ $K \mid J \in \mathbf{f}(D)$ and $J \subseteq I\}$ for each $I \in \mathbf{F}(D)$. Then the map $*_{a}$ : $\mathbf{F}(D) \rightarrow \mathbf{F}(D)$, given by $I \mapsto I^{* a}$, is an e.a.b. star operation of finite character on $D$ [10, Proposition 4.5 and Corollary 5.2]. It is known that $K r(D, *)=K r\left(D, *_{a}\right)$ and $I^{*_{a}}=\operatorname{IKr}(D, *) \cap K=\cap\left\{I V_{\beta} \mid V_{\beta}\right.$ is a *valuation overring of $D\}$ for each $I \in \mathbf{F}(D)$ [10]; hence $*_{c} \leq *_{a}$ since *-valuation overrings are $*$-linked, and so $\operatorname{Kr}\left(D, *_{c}\right) \subseteq \operatorname{Kr}\left(D, *_{a}\right)$.

Proposition 3. If $D$ is $*$-integrally closed, then $\operatorname{Kr}\left(D, *_{c}\right)=\operatorname{Kr}\left(D, *_{a}\right)$ if and only if each $*$-linked valuation overring of $D$ is a $*$-valuation overring. In this case, $*_{c}=*_{a}$.

Proof. $(\Rightarrow)$ Let $V$ be a $*$-linked valuation overring of $D$ that is not a $*$-valuation overring. Then there exists a $J \in \mathbf{f}(D)$ such that $J^{*} \nsubseteq$ $J V$. So $J V \subsetneq J^{*} V$, and hence $J^{*_{c}}=\cap\left\{J V_{\alpha} \mid V_{\alpha}\right.$ is a $*$-linked valuation overring of $D\} \subsetneq \cap_{\alpha} J^{*} V_{\alpha} \subseteq \cap\left\{J^{*} V_{\beta} \mid V_{\beta}\right.$ is a $*$-valuation overring of $D\}=\cap_{\beta} J V_{\beta}=J^{*_{a}}$. Thus $\operatorname{Kr}\left(D, *_{c}\right) \subsetneq \operatorname{Kr}\left(D, *_{a}\right)$ [13, Theorem 32.7]. $(\Leftarrow)$ Conversely, assume that each $*$-linked valuation overring of $D$ is a $*$-valuation overring. Then $I^{*_{c}}=I^{*_{a}}$ for all $I \in \mathbf{F}(D)$, and thus $*_{c}=*_{a}$ and $K r\left(D, *_{c}\right)=K r\left(D, *_{a}\right)$.

\section{A new characterization of $*$-quasi-Prüfer domains}

Let $D$ be an integral domain with quotient field $K, \bar{D}$ be the integral closure of $D$ in $K, X$ be an indeterminate over $D$, and $D[X]$ be the polynomial ring over $D$. Let $*$ be a star operation on $D$ and $N_{*}=\{f \in$ $\left.D[X] \mid c(f)^{*}=D\right\}$.

It is clear that $D$ is a $*_{f}$-quasi-Prüfer domain if and only if $c(Q)^{*_{f}}=D$ for each upper to zero $Q$ in $D[X]$. In particular, a $t$-quasi-Prüfer domain is exactly the same as the notion of a UMT-domain [15, Theorem 1.4]. Also, as in [8, page 210], we say that $D$ is a quasi-Prüfer domain if for each prime ideal $P$ of $D$, if $Q$ is a prime ideal of $D[X]$ with $Q \subseteq P D[X]$, then $Q=(Q \cap D) D[X]$. Hence $d$-quasi-Prüfer domains are just the quasi-Prüfer domains [5, Theorem 1.1]. It is known that a $*_{f}$-quasiPrüfer domain is a UMT-domain (Lemma $4((1) \Rightarrow(5))$. For useful characterizations of UMT-domains, see [7]. 
We next recall some characterizations of $*$-quasi-Prüfer domains, which are essential in the proof of the main result (Theorem 5) of this paper.

LEMma 4. The following statements are equivalent for a star operation $*$ on $D$.

(1) $D$ is a $*_{f}$-quasi-Prüfer-domain.

(2) The integral closure of $D[X]_{N_{*}}$ is a Prüfer domain.

(3) $D[X]_{N_{*}}$ is a quasi-Prüfer domain.

(4) $D_{P}$ is a quasi-Prüfer domain for each maximal $*_{f}$-ideal $P$ of $D$.

(5) $D$ is a UMT-domain and each maximal $*_{f}$-ideal of $D$ is a $t$-ideal.

(6) For each $0 \neq f \in D[X]$, there is a $0 \neq g \in K[X]$ such that $c_{D}(f g)^{*}=D$.

Proof. (1) $\Leftrightarrow(2) \Leftrightarrow(3) \Leftrightarrow(4) \Leftrightarrow(5)[5$, Theorem 2.16].

$(1) \Rightarrow(6)$ Let $f=f_{1}^{e_{1}} \cdots f_{k}^{e_{k}}$, where $f_{i} \in K[X], f_{i} K[X]$ is a prime, and $f_{i} K[X] \neq f_{j} K[X]$ for $i \neq j$. Then $f K[X] \cap D[X]=\left(f_{1}^{e_{1}} K[X] \cap\right.$ $\left.\cdots \cap f_{k}^{e_{k}} K[X]\right) \cap D[X]=\left(f_{1}^{e_{1}} K[X] \cap D[X]\right) \cap \cdots \cap\left(f_{k}^{e_{k}} K[X] \cap D[X]\right)$. Note that $f_{i} K[X] \cap D[X]$ is an upper to zero in $D[X]$; so there is a $0 \neq g_{i} \in K[X]$ such that $c_{D}\left(f_{i} g_{i}\right)^{*}=D$ by the definition of a $*_{f}$-quasiPrüfer domain. Clearly, $c_{D}\left(f_{i}^{e_{i}} g_{i}^{e_{i}}\right)^{*}=D$. Hence if we set $g=g_{1}^{e_{1}} \cdots g_{k}^{e_{k}}$, then $c_{D}(f g)^{*}=\left(c_{D}\left(f_{1}^{e_{1}} g_{1}^{e_{1}}\right) \cdots c_{D}\left(f_{k}^{e_{k}} g_{k}^{e_{k}}\right)\right)^{*}=D$.

$(6) \Rightarrow(1)$ Let $Q$ be an upper to zero in $D[X]$. Then $Q=f K[X] \cap D[X]$ for some $0 \neq f \in D[X]$ and $f$ irreducible in $K[X]$, and by (6), there is a $0 \neq g \in K[X]$ such that $c(f g)^{*}=D$. Clearly, $f g \in Q$. Thus $D$ is $*_{f}$-quasi-Prüfer.

Obviously, $\bar{D}[X]_{N_{*}}$ is the integral closure of $D[X]_{N_{*}}$; so $D$ is a $*_{f^{-}}$ quasi-Prüfer domain if and only if $\bar{D}[X]_{N_{*}}$ is a Prüfer domain by Lemma $4((1) \Leftrightarrow(2))$. We are now ready to prove the main result of this paper, which gives a new characterization of $*_{f}$-quasi-Prüfer domains including UMT-domains.

Theorem 5. Let $*$ be a star operation on $D$ and $K r\left(D, *_{w}\right)$ be as in Lemma 2. Then $D$ is a $*_{f}$-quasi-Prüfer domain if and only if $\bar{D}[X]_{N_{*}}=$ $\operatorname{Kr}\left(D, *_{w}\right)$.

Proof. $(\Rightarrow)$ We first note that if $D$ is $*_{f}$-quasi-Prüfer, then $D$ is a UMT-domain and $*_{w}=w$ by Lemma $4((1) \Rightarrow(5))$; so $N_{*}=N_{v}$. For convenience, we let $R=D^{\left[{ }^{*} w\right]}$.

Let $N_{v}(R)=\left\{f \in R[X] \mid c_{R}(f)^{v}=R\right\}$. Then $R$ is a $\mathrm{P} v \mathrm{MD}$ and $\bar{D}[X]_{N_{v}}=R[X]_{N_{*}}=R[X]_{N_{v}(R)}\left[6\right.$, Theorem 2.6]. Hence $R[X]_{N_{v}(R)}$ is a 
Bezout domain [16, Theorem 3.7], and thus each overring of $R[X]_{N_{v}(R)}$ is a quotient ring of $R[X]_{N_{v}(R)}\left[13\right.$, Theorem 27.5]. Note that $D[X]_{N_{*}} \subseteq$ $K r\left(D, *_{w}\right)$ and $K r\left(D, *_{w}\right)$ is integrally closed; so $\bar{D}[X]_{N_{*}} \subseteq \operatorname{Kr}\left(D, *_{w}\right)$. Thus $K r\left(D, *_{w}\right)$ is a quotient ring of $R[X]_{N_{v}(R)}$ (and hence of $R[X]$ ).

Let $S=\left\{f \in R[X] \mid \frac{1}{f} \in K r\left(D, *_{w}\right)\right\}$. Clearly, $K r\left(D, *_{w}\right)=R[X]_{S}$, and hence $f \in S$ if and only if there exists an $0 \neq h \in D[X]$ with $c_{D}(h)^{* w} \subseteq\left(c_{D}(f) c_{D}(h)\right)^{* w}$. Since $D$ is $*_{f}$-quasi-Prüfer, there exists a $0 \neq g \in K[X]$ such that $c_{D}(h g)^{* w}=D$ by Lemma 4 ; hence $c_{D}(h g) \subseteq$ $\left(c_{D}(h) c_{D}(g)\right)^{* w} \subseteq\left(c_{D}(f) c_{D}(h) c_{D}(g)\right)^{* w}$. Also, since $R$ is a PvMD and $N_{*} \subseteq N_{v}(R)\left[3\right.$, Theorem 4.1], we have $\left(c_{R}(h) c_{R}(g)\right)^{w}=c_{R}(h g)^{w}=R$. Hence by [3, Lemma 2.3],

$$
\begin{aligned}
c_{D}(h g) & \subseteq\left(c_{D}(f) c_{D}(h) c_{D}(g)\right)^{* w} \\
& =\left(c_{D}(f) c_{D}(h) c_{D}(g)\right) D[X]_{N_{*}} \cap K \\
& \subseteq\left(c_{R}(f) c_{R}(h) c_{R}(g)\right) R[X]_{N_{v}(R)} \cap K \\
& =\left(c_{R}(f) c_{R}(h) c_{R}(g)\right)^{w} \\
& =\left(c_{R}(f) c_{R}(h g)\right)^{w} \\
& =c_{R}(f)^{w}
\end{aligned}
$$

so $R=c_{R}(h g)^{v}=\left(c_{D}(h g) R\right)^{v} \subseteq c_{R}(f)^{v} \subseteq R$. Hence $c_{R}(f)^{v}=R$

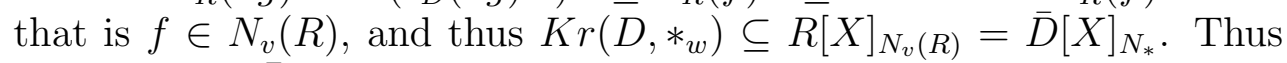
$\operatorname{Kr}\left(D, *_{w}\right)=\bar{D}[X]_{N_{*}}$.

$(\Leftarrow)$ Note that $\bar{D}[X]_{N_{*}}$ is the integral closure of $D[X]_{N_{*}}$ and $\operatorname{Kr}\left(D, *_{w}\right)$ is a Bezout domain. Thus $D$ is a $*_{f}$-quasi-Prüfer domain by Lemma $4((3)$ $\Rightarrow(1))$.

Recall that $d_{w}=d$ and $v_{w}=w$; so the following two corollaries are immediate consequences of Theorem 5 .

Corollary 6. $D$ is a quasi-Prüfer domain if and only if $\bar{D}(X)=$ $\operatorname{Kr}(D, d)$.

Corollary 7. D is a UMT-domain if and only if $\bar{D}[X]_{N_{v}}=\operatorname{Kr}(D, w)$.

It is known that $D$ is a $\mathrm{P} * \mathrm{MD}$ if and only if $D$ is an integrally closed $*_{f}$-quasi-Prüfer domain. Also, $D[X]_{N_{*}} \cap K=D$. Hence by Theorem 5 , we have

Corollary 8. ([9, Theorem 3.1]) $D$ is a $P * M D$ if and only if $D[X]_{N_{*}}=$ $\operatorname{Kr}\left(D, *_{w}\right)$.

Corollary 9. $D$ is a Prüfer domain if and only if $D(X)=K r(D, d)$. 
Acknowledgements. The author would like to thank the referees for their several valuable comments and suggestions.

\section{References}

[1] D.D. Anderson and S.J. Cook, Two star operations and their induced lattices, Comm. Algebra 28 (2000), 2461-2475.

[2] J.T. Arnold, On the ideal theory of the Kronecker function ring and the domain $D(X)$, Canad. J. Math. 21 (1969), 558-563.

[3] G.W. Chang, *-Noetherian domains and the ring $D[X]_{N_{*}}$, J. Algebra 297 (2006), 216-233.

[4] G.W. Chang, Prüfer *-multiplication domains, Nagata rings, and Kronecker function rings, J. Algebra 319 (2008), 309-319.

[5] G.W. Chang and M. Fontana, Upper to zero in polynomial rings and Prüfer-like domains, Comm. Algebra 37 (2009), 164-192.

[6] G.W. Chang and M. Zafrullah, The w-integral closure of integral domains, J. Algebra 259 (2006), 195-210.

[7] M. Fontana, S. Gabelli, and E. Houston, UMT-domains and domains with Prüfer integral closure, Comm. Algebra 26 (1998), 1017-1039.

[8] M. Fontana, J. Huckaba, and I. Papick, Prüfer domains, Marcel Dekker, 1997.

[9] M. Fontana, P. Jara, and E. Santos, Prüfer *-multiplication domains and semistar operations, J. Algebra Appl. 2 (2003), 1-30.

[10] M. Fontana and K.A. Loper, Kronecker function rings: a general approach, in Ideal Theoretic Methods in Commutative Algebra, Lecture Notes in Pure Appl. Math., Marcel Dekker, 220 (2001), 189-205.

[11] M. Fontana and K.A. Loper, Nagata rings, Kronecker function rings and related semistar operations, Comm. Algebra 31 (2003), 4775-4801.

[12] M. Fontana and K.A. Loper, A historical overview of Kronecker function rings, Nagata rings, and related star and semistar operations, in: J.W. Brewer, S. Glaz, W.J. Heinzer, B.M. Olberding (Eds.), Multiplicative Ideal Theory in Commutative Algebra. A Tribute to the Work of Robert Gilmer, Springer, 2006, pp. 169-187.

[13] R. Gilmer, Multiplicative Ideal Theory, Dekker, New York, 1972.

[14] E. Houston, S. Malik, and J. Mott, Characterizations of *-multiplication domains, Canad. Math. Bull. 27 (1984), 48-52.

[15] E. Houston and M. Zafrullah, On t-invertibility, II, Comm. Algebra 17 (1989), 1955-1969.

[16] B.G. Kang, Prüfer v-multiplication domains and the ring $R[X]_{N_{v}}$, J. Algebra 123 (1989), 151-170.

[17] A. Okabe and R. Matsuda, Star operations and generalized integral closures, Bull. Fac. Sci., Ibaraki Univ. 24 (1992), 7-13. 
Department of Mathematics

University of Incheon

Incheon 406-772, Korea

E-mail: whan@incheon.ac.kr 